\title{
"MUCHO RUIDO Y POCAS NUECES": LA DIPLOMACIA CULTURAL Y LA IMAGEN-PAÍS DE MÉXICO EN EL SEXENIO DE ENRIQUE PEÑA NIETO
}

\author{
"MUCH ADO ABOUT NOTHING": CULTURAL \\ DIPLOMACY AND THE COUNTRY IMAGE OF MEXICO \\ DURING THE PRESIDENCY OF ENRIQUE PEÑA NIETO
}

\section{"BEAUCOUP DE BRUIT POUR RIEN": LA DIPLOMATIE CULTURELLE ET L'IMAGE DU MEXIQUE SOUS LE GOUVERNEMENT D'ENRIQUE PEÑA NIETO}

\author{
César Villanueva Rivas \\ Universidad Iberoamericana \\ cesar.villanueva@uia.mx
}

Resumen: Este artículo analiza cómo la diplomacia cultural del gobierno de Enrique Peña Nieto tuvo presencia en las relaciones internacionales de México, pero con un desorden conceptual y de manera irrelevante en tanto herramienta de política exterior. La imagen de México en el extranjero tuvo un buen inicio con el llamado Mexican Moment, pero se desplomó después de los hechos asociados con la corrupción y la escalada de crímenes, específicamente con el caso de Ayotzinapa de 2014. Por su parte, el presupuesto destinado a la diplomacia cultural fue una cifra nominal y decorativa ejercida desde la Dirección General de Cooperación Educativa y Cultural de la Amexcid, cuyo desempeño fue irrelevante debido a la falta de coordinación y redundancia de las iniciativas de las secretarías de Cultura, Economía, Turismo y la Presidencia de la República.

Palabras clave: diplomacia cultural; diplomacia pública; imagen-país; cooperación cultural; política exterior; México.

ABstRACT: This article analyzes how the cultural diplomacy of the government of Enrique Peña Nieto had a presence in the international relations of Mexico, but suffered from a conceptual disorder and irrelevance as a tool of foreign policy. The image of Mexico abroad had a good start with the so-called 
Mexican Moment, but collapsed following corruption-related events and the escalation of criminal activity, and specifically the Ayotzinapa case of 2014. Meanwhile, the budget for cultural diplomacy was merely a nominal and decorative figure exercised by the Department for Educational and Cultural Cooperation of Amexcid, whose expenditure was made irrelevant by the lack of coordination and redundancy of the initiatives of the Ministries of Culture, Economy, Tourism and the Presidency of the Republic.

Keywords: cultural diplomacy; public diplomacy; country image; cultural cooperation; foreign policy; Mexico.

\section{Traducción de Gonzalo Celorio Morayta}

RÉsumé: L'article analyse de quelle façon la diplomatie culturelle du gouvernement d'Enrique Peña Nieto a été présente dans les rapports du Mexique avec l'extérieur, malgré un certain désordre dans les idées et quelques faiblesses en tant qu'outil de la politique étrangère. L'image internationale du pays a fait un bon début lors du Mexico's moment, mais elle s'est brisée à cause des scandales de corruption et de la montée du crime, mise en évidence par l'affaire Ayotzinapa en 2014. De plus, le budget destiné à la diplomatie culturelle a été plus symbolique que réel, tandis que les travaux de la Direction générale de la coopération éducative et culturelle (qui fait partie de l'Agence mexicaine de coopération internationale pour le développement, Amexcid) n'ont pratiquement pas eu d'effets, à cause de la duplication d'efforts et du manque de coordination entre le bureau du président de la République et les secrétariats à la Culture, à l'Économie et au Tourisme.

Mots clefs: diplomatie culturelle; diplomatie publique; image du pays; coopération culturelle; politique étrangère; Mexique.

Traducción de Bernardo Mabire

Fecha de recepción: diciembre de 2018

Fecha de aceptación: febrero de 2019 
$\mathrm{E}$ L campo de la diplomacia cultural ha evolucionado enormemente los últimos años, tanto en los aspectos conceptuales como en los empíricos y, sin duda, en los referidos al desarrollo tecnológico. El cambio se inicia en el terreno epistémico. La naturaleza de los conceptos se ha transformado en tiempos recientes, generando un rasgo peculiar y pragmático: la hibridación conceptual. La diplomacia cultural como escenario de acciones hacia el exterior, entendida en términos de la primera década del milenio, se ha modificado para incorporar tres visiones distintivas: lo "interméstico" (o, si se prefiere, "glocal"), el "infotainment" de los contenidos y, más radical y específico, la invención del mix de la diplomacia cultural y pública en lo que denominamos "culpub diplomacy". En esta última, la diplomacia cultural abarca acciones de política exterior enfocadas en la difusión de la identidad nacional, mediante valores, tradiciones, creencias y arte, en tanto que la pública está dirigida a realizar acciones de gestión de la información, así como políticas y estrategias de comunicación cuyo objetivo es modificar y mejorar la opinión de las audiencias nacionales y extranjeras. Existen ejemplos muy definidos de política exterior en los cuales predomina la diplomacia pública sobre la cultural y viceversa. En este sentido, la política exterior de los países anglosajones está claramente afianzada en una estrategia de diplomacia pública a la par que, por otro lado, algunos Estados se orientan más hacia la proyección internacional de su identidad nacional, como Francia, que se deriva en lo que se entiende como diplomacia cultural. Sin embargo, debido a la limitación de recursos, al desarrollo de nuevas tecnologías y al surgimiento de nuevos actores, ha emergido una hibridación entre ambas categorías (la diplomacia pública y cultural) para alcanzar los principios de política exterior de los diferentes países.

En relación con los objetivos, el giro ha sido hacia un "transversalismo" metodológico binacional y multilateral, buscando metas más cosmopolitas ancladas en bienes públicos globales, a través de una variación de los intereses hacia la in- 
terdireccionalidad estatal, dejando atrás el aburrido unidireccionalismo de la promoción y propaganda cultural. Finalmente, en el plano tecnológico, los tres ejes del cambio radical residen en la intensificación del uso de las redes sociodigitales, el uso abierto de centros digitales con información masiva y la participación ampliada de sectores sociales anteriormente excluidos de esta práctica diplomática, como las diásporas. ${ }^{1}$

Para efectos prácticos, la diplomacia cultural de México en el período de Enrique Peña Nieto experimentó sólo algunos de estos grandes cambios. En este campo, se continúa operando de manera muy semejante a la del último tramo del siglo xx, con una imagen propia claramente tradicional, con objetivos predecibles y con un uso muy limitado de la tecnología disponible. Un ejemplo claro es el abandono que sufrió el circuito de doce institutos culturales de México en el exterior, que penosamente siguen funcionando sin ninguna brújula ni apoyos reales para cumplir su misión. El problema central no se refiere únicamente a los recursos económicos disponibles, sino más bien a la manera tan conservadora como se construyó esta área y se mantuvo el estereotipo de ser meramente un elemento decorativo de la diplomacia formal.

Por otro lado, la imagen-país de México en el exterior es una ecuación que obedece a diversos factores y, ciertamente, tiene en la diplomacia cultural un aliado, pero la diplomacia pública es un resorte de mayor alcance para su proyección. En paralelo, las oficinas de turismo, de economía, de cultura y, sin lugar a dudas, de marca país, tienen muchos elementos que aportar para una mejor percepción de México en el exterior. Sin embargo, por razones asociadas con la violencia proveniente de fuentes como las industrias negras del narcotráfico, el crimen de alto impacto y la corrupción institucio-

${ }^{1}$ Eduardo Tadeo, "Public Diplomacy, Soft Power and Language: The Case of the Korean Language in Mexico City", Journal of Contemporary Eastern Asia, 17 (2018), pp. 27-49. 
nal, esta imagen ha sido construida predominantemente de manera negativa en los medios internacionales.

El presente artículo se propone entender las maneras en que el gobierno federal y, en especial, la Secretaría de Relaciones Exteriores pusieron en práctica las nociones de diplomacia cultural e imagen de México en el sexenio de 2013 a 2018. En primer lugar, se hace una descripción del desorden conceptual y operativo en cómo el gobierno de Enrique Peña Nieto (EPN) definió el campo de acción de la diplomacia cultural.

\section{VISIÓN, MISIÓN Y OBJETIVOS DE LA DIPLOMACIA Cultural de MÉxico}

La misión y visión de la diplomacia cultural de México quedaron establecidas desde enero de 2009, cuando se anuncia el cambio de denominación y de adscripción del área de la SRE, como paso previo al anuncio de abril de 2010 que formalizaba dicho cambio en la Ley de Cooperación Internacional para el Desarrollo. ${ }^{2}$ Estos cambios se vieron reflejados en el Presupuesto de Egresos de 2011, que, de facto, formaliza la creación de la Agencia Mexicana de Cooperación Internacional para el Desarrollo (Amexcid) como un órgano desconcentrado de la SRE, dotado de autonomía técnica y de gestión. El problema organizacional y de conceptualización proviene de las discusiones previas a la formalización de la agencia, cuando se definieron los objetivos, la visión y la misión propios del área. Lo que la diplomacia cultural vive ahora es, en parte, un conjunto de consecuencias de tales decisiones, que se reflejan de manera precisa en el Reglamento Interior de la Secretaría de Relaciones Exteriores, específicamente en el artículo 37. En éste, se establecen

${ }^{2}$ César Villanueva, "Crónica de un declive anunciado: la diplomacia cultural en el sexenio de Felipe Calderón”, Foro Internacional, LIII, núms. 3-4 (2013), pp. 845-872. 
de manera precisa las funciones de la Dirección General de Cooperación Educativa y Cultural (DGCEC), que en síntesis pueden describirse como la elaboración de los lineamientos de la diplomacia cultural, dividida en cooperación y promoción propiamente dichas, así como el establecimiento de las coordinaciones y enlaces necesarios con otras áreas del gobierno para proveer información, negociar y ejecutar acciones tendientes a promover la imagen cultural de México en el exterior. ${ }^{3}$

Es importante señalar que la misión de la DGCEC quedó establecida así: "Facilitar a la cancillería y a las representaciones de México en el exterior el manejo eficiente de la cooperación educativa y cultural de acuerdo con los objetivos de política exterior que fije para tal fin el gobierno de México”, mientras que la visión se especificó de la siguiente manera: "Ser una unidad administrativa eficiente en la defensa, difusión y promoción de la cultura mexicana en el exterior a fin de preservar y fortalecer la posición de México en el ámbito internacional”. De igual manera, los objetivos de la diplomacia cultural mexicana son dos y se definieron de la siguiente forma: ${ }^{4}$

- Coordinar la promoción de los intereses de México en el mundo, a través de la cooperación educativa y cultural y la promoción de la cultura, y fortalecer las capacidades nacionales en materia educativa por medio del intercambio y su vinculación internacional.

- Impulsar y proyectar la imagen positiva de México en el mundo a través de la educación y la cultura, en coordinación con las instituciones mexicanas, las embajadas, consula-

${ }^{3}$ Secretaría de Relaciones Exteriores, SRE, Agencia Mexicana de Cooperación Internacional para el Desarrollo, Amexcid, "Manual de Organización de la Dirección General de Cooperación Educativa y Cultural, México, SRE, 2012, https://sre.gob.mx/images/stories/docnormateca/ manadmin/2012/modgcec.pdf

${ }^{4}$ Loc. cit. 
dos y delegaciones mexicanas en el exterior, además de los sectores privado y social.

¿Qué podemos aprender de este ordenamiento legal y administrativo? Algo que llama la atención es que la diplomacia cultural no aparece como tal en ninguno de los documentos analizados. La referencia específica es a las "acciones de promoción y cooperación”, que deben entenderse como una dicotomía de la acción misma de la diplomacia cultural. Este dato no es menor, dado que los documentos oficiales no aportan de manera específica la claridad conceptual necesaria para darle sustancia a la acción diplomática y, como veremos más adelante, las consecuencias de ello son importantes.

\section{EL DESORDEN CONCEPTUAL Y ORGANIZACIONAL ENTRE DIPLOMACIA PÚBLICA Y CULTURAL}

En años recientes, los debates especializados y las prácticas disponibles nos obligan a cambiar de modo sustancial la forma en que se concibe ${ }^{5}$ y hace operativa la diplomacia cultural en el mundo. ${ }^{6}$ En el sexenio que analizamos, las diplomacias pública y cultural se integran como un objetivo del Plan Nacional de Desarrollo, específicamente para "promover el valor de México en el mundo mediante la difusión económica, turística y cultural". ${ }^{7}$ Dicho objetivo va acompañado de estrategias precisas para alcanzarlo, entre las que sobresale la

${ }^{5}$ Cynthia Schneider, "Cultural diplomacy: hard to define, but you'd know if you saw it", Brown Journal of World Affairs, vol. 13m, núm. 1 (2006), pp. 191-203.

${ }^{6}$ Dimitra Kizlari y Kalliopi Fouseki, "The Mechanics of Cultural Diplomacy: A Comparative Case Study Analysis from the European Context", The Journal of Arts Management Law and Society, vol. 48, núm. 2 (2017), pp. 133-147.

${ }^{7}$ Plan Nacional de Desarrollo 2013-2018, Diario Oficial de la Federación, 20 de mayo de 2013, objetivo 5.2, https://www.dof.gob.mx/nota_detalle_popup.php?codigo $=5299465$ 
de "definir agendas en materia de diplomacia pública y cultural que permitan mejorar la imagen de México en el exterior, lo cual incrementará los flujos de comercio, inversión y turismo para elevar y democratizar la productividad a nivel regional y sectorial", con sus respectivas líneas de acción, que quedaron delineadas de la siguiente forma:

- Impulsar la imagen de México en el exterior mediante una amplia estrategia de diplomacia pública y cultural.

- Promover que los mexicanos en el exterior contribuyan a la promoción de la imagen de México.

- Emplear la cultura como instrumento para la proyección de México en el mundo, con base en las fortalezas del país.

- Aprovechar los bienes culturales, entre ellos la lengua española y los productos de las industrias creativas, como instrumentos de intercambio diplomático, diálogo y cooperación.

- Impulsar los vínculos de los sectores cultural, científico y educativo mexicano con sus similares en Latinoamérica y otras regiones del mundo. ${ }^{8}$

De lo anterior se derivan dos aspectos importantes. El primero es que el Plan Nacional de Desarrollo tendía a una visión multidimensional del objetivo general de "promover el valor de México en el mundo". Éste es un punto de partida bien estructurado que, sin embargo, mantiene un enfoque tradicional ("yo te cuento mi historia"). Lo siguiente a considerar es la intervención de la SRE y su brazo operativo, la Amexcid. Lo primero y más obvio es la manera en que ésta incorpora el tema diplomático en su corpus operativo, así como las áreas intrasecretariales que participan indirectamente en estos temas: diversas subsecretarías, el Instituto de los Mexicanos en el Exterior (IME), la Dirección de Comunicación Social, la Dirección General de Vinculación con las Organizaciones de la Sociedad Civil y, por supuesto, las em-

${ }^{8}$ Ibid., Estrategia, 5.2.2. 
bajadas y consulados de México en el exterior, así como el Instituto Matías Romero.

En un segundo nivel, es pertinente revisar cómo se asocian estos rubros con otras áreas del gobierno (véase la figura 1), principalmente la hoy Secretaría de Cultura (SC) -que hasta 2016 fuera el Consejo Nacional para la Cultura y las Artes-y la Secretaría de Economía, en especial ProMéxico y el Consejo de Promoción Turística de México (СРTM). En un tercer nivel no menos importante, se incorporan, también en el ámbito federal, la Coordinación de Marca País y Medios Internacionales de la Presidencia de la República y la Secretaría de Turismo. En un cuarto nivel, de orden estatal, existen las diversas oficinas de asuntos internacionales de las entidades de la Federación, que atienden temas asociados con su promoción y la cooperación a nivel federal. Finalmente, se tienen diversas agencias públicas o privadas que ejercen actividades de promoción cultural e imagen en el exterior, con una agenda muy específica, como es el caso de la Universidad Nacional Autónoma de México, el Instituto Politécnico Nacional, la Universidad de Guadalajara y otras instituciones similares con presencia e interés en el ámbito académico internacional.

En cuanto a la diplomacia pública, como dice la conocida expresión tomada de una obra shakesperiana: "Mucho ruido y pocas nueces". En realidad, la SRE no sabe hacer diplomacia pública, al menos de la forma como se concibe en aquellos países que han desarrollado ampliamente dicho concepto. ${ }^{9}$ Lo que la SRE practica es un conjunto de acciones casuísticas, de mayor o menor impacto, con un costo muy elevado y caracterizadas por la improvisación y el oportunismo económico. Los beneficios son francamente cuestionables respecto a los objetivos de la política exterior de México. En la sección dedicada al presupuesto, en este artículo, esto se vuelve más evidente.

${ }^{9}$ Para consultar una de las mejores referencias sobre diplomacia pública, véase United States Advisory Commission on Public Diplomacy, "2017 Comprehensive Annual Report on Public Diplomacy \& International Broadcasting", eEuu, Departamento de Estado, 2017, https://www. state.gov/documents/organization/274950.pdf 


\section{Figura 1}

\section{Áreas responsables de la Diplomacia Cultural y la Imagen País}

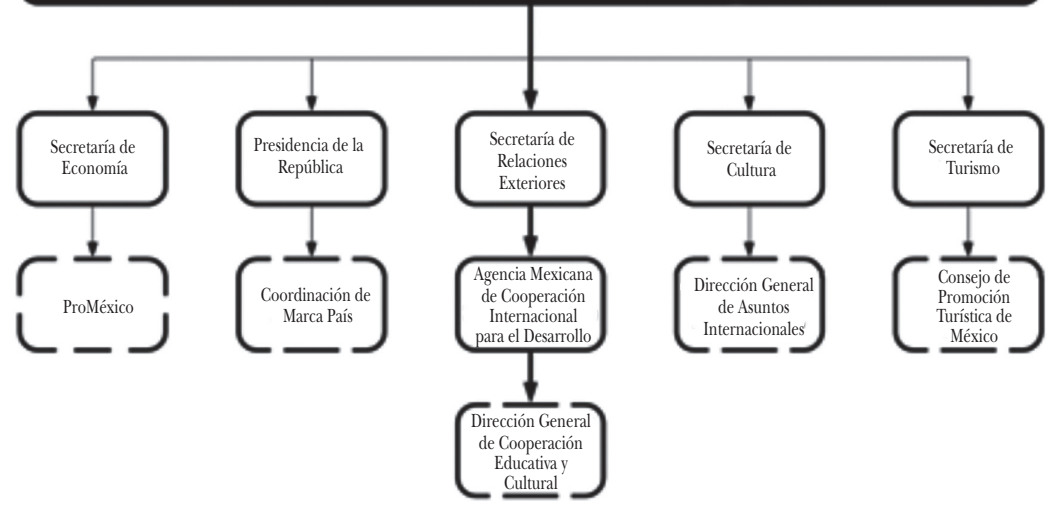

Fuente: elaboración propia con base en el organigrama de las dependencias gubernamentales referidas.

Regresando a la figura 1, podría pensarse que la Presidencia de la República conducía el tema de la diplomacia pública, asociándolo a la imagen de México y, por tanto, como una prioridad del Estado. Nada más lejano a la verdad. La Coordinación de Marca País y Medios Internacionales, creada en abril de 2015, tuvo una enorme discrecionalidad y falta de transparencia en las acciones que realizó, así como en los presupuestos que ejercía. Emanada de una reorganización interna de la Presidencia, tomó el lugar de la Coordinación de Estrategia y Mensaje Gubernamental y de la Coordinación de Vinculación, cuyas funciones fueron reasignadas a otras unidades administrativas de la oficina presidencial. En efecto, de acuerdo con el decreto respectivo, corresponde a esa nueva instancia "participar en el diseño y aplicación de las estrategias de comunicación para fortalecer la imagen y percepción de México en el extranjero, en coordinación con las demás unidades competentes de la Oficina de la Presidencia, así como de las dependencias y entidades de la administración pública federal”. Se añade que también es responsable 
de colaborar con los medios de comunicación de otros países, así como de compilar "la información publicada y difundida en los medios de comunicación del extranjero", entre otras actividades. ${ }^{10} \mathrm{En}$ realidad, fueron pocos los resultados visibles de la gestión de su primer director, Paulo Carreño King, a quien le sucedió Carla Sánchez Armas García, que compartió con su antecesor la práctica de no tener una rendición de cuentas transparente.

Se puede decir que la diplomacia pública también se atendió en el gobierno de EPN desde la Secretaría de Turismo, específicamente desde el CPTM. Su misión específica es la de "promocionar integral y competitivamente a México, con sus productos y destinos, en los mercados nacionales e internacionales, a través del trabajo conjunto entre todos los actores de la actividad turística" ${ }^{11}$ Lo más reconocible en este campo, pero no necesariamente distinguible del turismo propiamente dicho, ha sido la campaña "Mexico Live it to Believe it", con resultados que lucen favorables para la atracción turística de extranjeros. Asimismo, esta área decidió organizar eventos de alto impacto para captar públicos externos que, de otra manera, no pondrían atención en México. Los cuatro eventos bandera de esta estrategia de diplomacia pública fueron, primero, la realización del Campeonato Mundial de Fórmula 1 en la Ciudad de México durante al menos cuatro años (de 2015 a 2018); segundo, el apoyo a las actividades del Año Dual México-Alemania (2016-2017); tercero, la celebración de un partido de futbol americano profesional con equipos de la National Football League (NFL), de EEUU, durante cuatro temporadas, en la Ciudad de México y, finalmente, la promoción de un Desfile del Día de Muertos, también en la capital del país, en ocasión del impacto doméstico que tuvieron los 19 minutos iniciales de la película Spectre, de 2015.

${ }^{10}$ Decreto por el que se reforman, adicionan y derogan diversas disposiciones del Reglamento de la Oficina de la Presidencia de la República, Diario Oficial de la Federación, 30 de abril de 2015, art. 12 bis.

${ }^{11}$ Consejo de Promoción Turística de México, Primer informe de logros - Programa Institucional 2014-2018, Ciudad de México, CPTM, 2014. 
Otra manifestación de diplomacia pública del gobierno federal ha sido la relacionada con ProMexico, dependiente de la Secretaría de Economía. Su misión es la de "promover la atracción de inversión extranjera directa y las exportaciones de productos y servicios, así como la internacionalización de empresas mexicanas, para contribuir al desarrollo económico y social del país y al fortalecimiento de la imagen de México como socio estratégico para hacer negocios". ${ }^{12}$ Entre sus objetivos, el número VI plantea la coordinación con la SRE para los temas de inversión y para coordinar las 49 Oficinas de Representación en el Exterior (oREx), presentes en 32 países, que a su vez, se coordinaron con las misiones diplomáticas de la cancillería para articular esfuerzos. La colaboración también se extendió a la Coordinación de Marca País y el CPTM, con los consecuentes traslapes de objetivos e intereses mutuos. ProMéxico fue responsable, entre otros rubros, de los pabellones mexicanos en ferias internacionales (específicamente, la Expo Milán 2015, en Italia, y la Expo Astaná 2017, en Kazajistán), de las "misiones de exportadores, importadores e inversionistas", de los "eventos de promoción internacionales" y de los "seminarios especializados para exportacion". Lo incomprensible son las duplicidades, gastos discrecionales y campañas que, en muchos sentidos, carecen de sensibilidad y proyección de una imagen adecuada de México en el exterior. ${ }^{13}$ Como lo ha documentado César Corona, "el gobierno federal gastó 485 millones de pesos en recursos públicos, a través del contrato $\mathrm{PM} / \mathrm{C} / 176 / 2014$, para que México participara en la Expo 2015 Milán”. ${ }^{14}$ Los resultados, más allá de lo anecdótico, parecen un catálogo de cómo hacer cosas malas que parezcan buenas, por la falta

12 ProMéxico, “¿Qué hacemos?”, México, Gobierno de la República, https://www.gob.mx/promexico/que-hacemos

${ }^{13}$ Nicholas Cull, "El futuro de la diplomacia pública: implicaciones para México”, Revista Mexicana de Política Exterior, 2012, núm. 96, pp. 45-73.

${ }^{14}$ César Corona, "1. Un proyecto de 485 millones de pesos sin pies ni cabeza" (blog personal), 17 de enero de 2106, https:/ / cesarcorona.exposure.co/intro-mexico-expo2015 
de transparencia, fallas de estrategia y dispendio. En contraste, la Expo Astaná resultó ser prácticamente gratuita para México, debido a la invitación específica del gobierno de Kazajistán. ${ }^{15}$ ¿Qué definió la estrategia de diplomacia pública de ProMexico estos años? Es una pregunta que no tiene respuesta fácil.

El TEMA DE LA GULTURA PARA LA POLÍTICA EXTERIOR DE MÉXICO

Resulta muy sorprendente conocer lo poco que los diplomáticos activos, en general, entienden de la cultura. Más complejo aún es instrumentar una política exterior desde donde apropiarse de la cultura como una herramienta diplomática. Por su naturaleza, el tema cultural convoca a diversas áreas del quehacer público. Es momento de establecer para este artículo, cómo se utilizó la cultura como una estratagema desde el gobierno federal, fundamentalmente desde la SRE y la sc. Como se puede observar en el cuadro 1, que recoge algunas acciones selectas, la cultura ha sido una herramienta de proyección de identidades mexicanas, con un sustrato histórico basado en las grandes civilizaciones antiguas, figuras célebres de la literatura y la pintura del siglo $\mathrm{xx}$, y que incluye algunos personajes vivos con una trayectoria reconocida en el campo de las artes. Esta línea discursiva no varía mucho de lo que se ha venido haciendo desde, al menos, 1990, cuando se estableció el canon propuesto por "México: Esplendores de treinta siglos", ${ }^{16}$ la exposición emblemática que proyectó al país hacia Norteamérica en el gobierno de Carlos Salinas de Gortari.

15 "ProMéxico. México participará en Expo Mundial Astaná 2017", ProMéxico, Comunicado 39/2017, 1 de junio de 2017, http://www.pro mexico.gob. $\mathrm{mx} / \mathrm{es} / \mathrm{mx} /$ comunicado-39-17

${ }^{16}$ Karina Olivares, Diplomacia cultural de México en el periodo 1988-1994: el caso de "México, Esplendores de Treinta Siglos", México, unam, 2005. 


\section{Cuadro 1 \\ Acciones culturales emblemáticas en el exterior (selección 2013-2018)}

\begin{tabular}{|c|c|c|}
\hline Año & Actividad & Perfil \\
\hline 2013 & $\begin{array}{l}\text { "México: A Revolution in Art, 1910-1940", exposición en la Royal } \\
\text { Academy of Arts de Londres. }\end{array}$ & Muy alto \\
\hline 2013 & $\begin{array}{l}\text { "Aztecs: Conquest and Glory", exposición en el Museum of New } \\
\text { Zeland Te Papa Tongarewa. }\end{array}$ & Medio \\
\hline 2014 & $\begin{array}{l}\text { Presentación del ciclo de cine mexicano "Una mirada a México", } \\
\text { en Addis Abeba, Etiopía. }\end{array}$ & Medio \\
\hline 2014 & $\begin{array}{l}\text { Conversación entre Elena Poniatowska y Jordi Soler, en el marco } \\
\text { de la entrega del Premio Cervantes, en el Instituto de México en } \\
\text { Madrid. }\end{array}$ & Medio \\
\hline 2015 & $\begin{array}{l}\text { Participación en la London Book Fair, en el marco del Año Dual } \\
\text { México Reino-Unido. }\end{array}$ & Medio \\
\hline 2015 & $\begin{array}{l}\text { "A Taste of México", evento culinario en el Instituto Cultural de } \\
\text { México en Washington, DC. }\end{array}$ & Bajo \\
\hline 2016 & $\begin{array}{l}\text { "Relato de una Negociación", exposición de Francis Alÿs, en el } \\
\text { Museo de Arte Latinoamericano de Buenos Aires. }\end{array}$ & Muy Alto \\
\hline 2016 & $\begin{array}{l}\text { Participación en el XV Festival Iberoamericano de Teatro de } \\
\text { Bogotá, como país invitado de honor. }\end{array}$ & Medio \\
\hline 2016 & $\begin{array}{l}\text { "Frida Kahlo - Sus fotos", exposición en el Museu da Imagem e do } \\
\text { Som, São Paulo. }\end{array}$ & Muy Alto \\
\hline 2016 & $\begin{array}{l}\text { Exposición de arte "Sergio Hernández, Tres Pasiones" y Códice } \\
\text { Hernandino-Mixteco, Labirinto della Masone, Fontanellato, } \\
\text { Parma. }\end{array}$ & Medio \\
\hline 2018 & $\begin{array}{l}\text { "Pintado en México 1700-1790: Pinxit Mexici", exposición en Los } \\
\text { Angeles County Museum of Art. }\end{array}$ & Alto \\
\hline 2018 & $\begin{array}{l}\text { Participación del chef Vicente Etchegaray en la presentación de La } \\
\text { Liste, compendio de los mejores restaurantes del mundo. }\end{array}$ & Bajo \\
\hline
\end{tabular}

Fuente: informes anuales de la Amexcid 2012-2018.

Como puede observarse en la gráfica 1, el volumen de acciones culturales (9746) tuvo fluctuaciones anuales que permiten observar los cambios experimentados por este indicador. El año con más acciones en el exterior fue 2017 (2113) y en el que menos hubo fue 2013 (662). Durante 2014, 2015 y 
2016 se presentó una relativa estabilidad en el número de acciones, pero en 2018 la cifra es casi de la mitad del año previo.

\section{GRÁFICA 1}

Acciones culturales en el exterior y presupuesto (2013-2018)

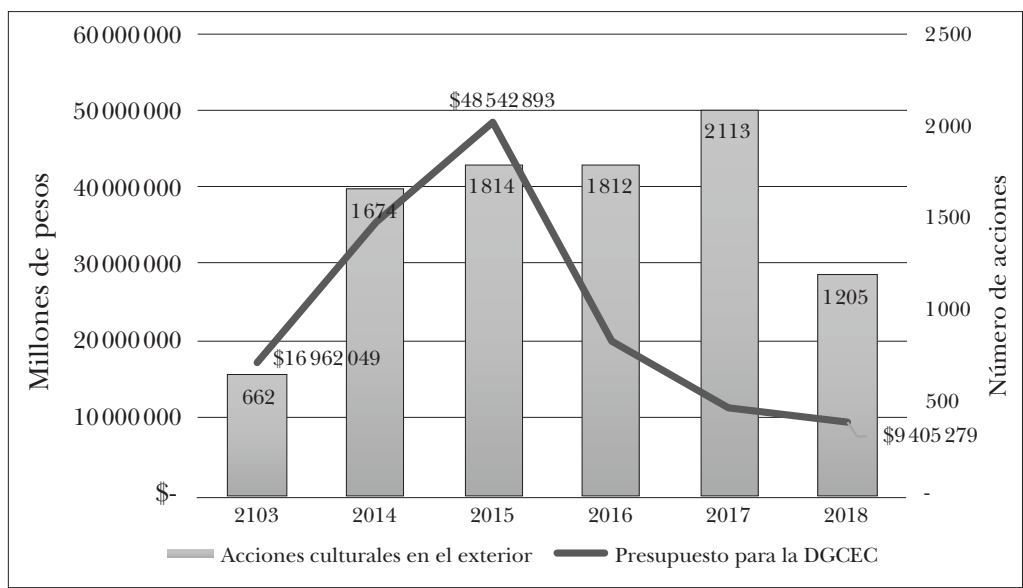

Fuente: Amexcid y SRE.

La explicación del número de acciones culturales en el exterior y las variaciones que muestra la gráfica 1 abarca tres aspectos. En 2013, el director de la Amexcid, Juan Manuel Valle Pereña, decidió mantener temporalmente a la embajadora Cecilia Jaber Breceda como titular de la DGCEC. Esto generó incertidumbre por la carencia de un proyecto sexenal específico para esta dirección y, al mismo tiempo, fue muestra del poco interés de Valle Pereña en esa área. Posteriormente, entraron tres personas a dirigir un proyecto con una ruta de navegación más o menos clara, ${ }^{17}$ y es entonces

17 En 2014, la Amexcid presentó el Programa de Cooperación Internacional para el Desarrollo 2014-2018, del que se derivó el plan estratégi- 
que la cantidad de iniciativas se estabiliza e, incluso, rebasa la histórica cantidad de 2000, en 2017. La caída en el número de acciones culturales en el año 2018 tuvo que ver con el abandono del proyecto por el canciller Luis Videgaray y, específicamente, del director de la Amexcid, Agustín GarcíaLópez Loaeza.

Como también puede apreciarse en la gráfica 1, no hay correlación entre presupuesto asignado y número de iniciativas o acciones, lo cual provoca enormes dudas sobre las metodologías aplicadas por la Amexcid. Así, en 2013 se consigna un presupuesto de 16.9 millones de pesos y la DGCEC reporta 662 acciones para ese periodo, es decir, el costo promedio anual por acción cultural internacional para la SRE fue de 25600 pesos. En 2015, el presupuesto se elevó a 48.5 millones de pesos, el triple que el primer año. A su vez, se reportó un total de 1814 acciones, también casi tres veces más que en 2013. Y, en 2015, cada acción cultural le costó a la SRE 26700 pesos, cifra similar a la de 2013. Esta constancia se pierde en los tres años siguientes, como se aprecia en la gráfica 1. Hay mucho menos presupuesto disponible para la DGCEC, pero el número de acciones se mantiene e, incluso, crece en 2017 , para caer en 2018. Este último año el presupuesto fue de 9.4 millones de pesos y se reportaron 1205 acciones, lo que equivale a un costo unitario de 7800 pesos.

En cuanto al tipo de actividades que se realizaron, es importante decir que siguieron predominando las artes visua-

co de diplomacia cultural para el periodo 2014- 2018 basado en los siguientes ejes de acción: "México, una potencia cultural", para promover contenidos estratégicos, generando un intercambio cultural incluyente y plural; "México, una necesidad en la agenda del mundo", con el objetivo de generar en la conciencia del mundo el reconocimiento y la demanda cultural mexicana contemporánea de una forma dinámica, y "México contemporáneo", enfocado en crear nuevos modelos de gestión a través de las tendencias actuales para materializar una política cultural de alto impacto. Véase Programa de Cooperación Internacional para el Desarrollo 2014-2018, Diario Oficial de la Federación, 30 de abril de 2014, http: / / www.dof.gob.mx/nota_detalle.php?codigo=5342827\&fecha=30/04/2014 
les $(51 \%)$, seguidas de los medios audiovisuales (20\%) y, en menor medida, las artes escénicas $(14 \%)$ y las conferencias y la literatura $(10 \%)$. Lo que esto nos dice es que se mantiene una visión tradicional del trabajo de la diplomacia cultural, tanto en las propuestas como en la práctica, y que los operadores no pudieron ir más allá de estos enfoques tradicionales. El sexenio anterior al de EPN siguió la misma ruta.

\section{GrÁFICA 2}

Tipo de actividades de diplomacia cultural de México en el exterior (2013-2018)

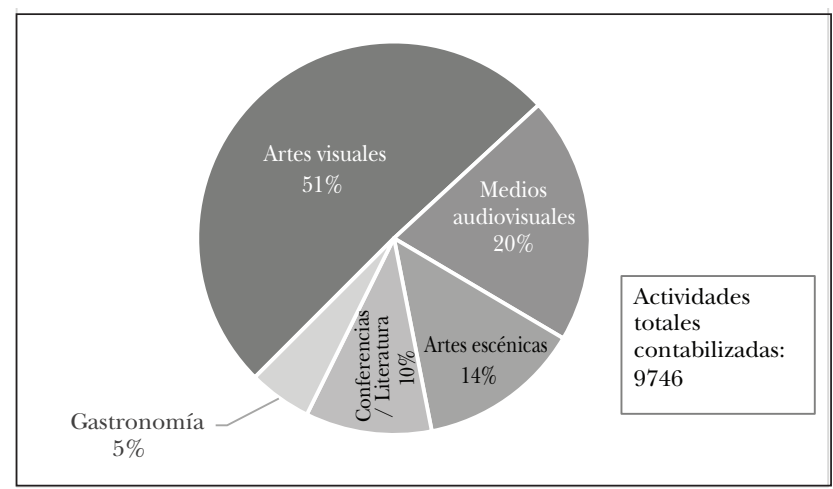

Fuente: informes anuales de la SRE.

De manera similar, la diplomacia cultural mexicana tuvo una actividad inercial en cuanto a las regiones destinatarias de las acciones de la SRE en la materia. Como es evidente en la gráfica 3, existe una continuidad geocultural con lo realizado, al menos, los últimos treinta años. Predomina Europa (36\%), seguida de América del Norte $(30 \%)$ y América Latina y el Caribe (20\%). Las regiones de Asia Pacífico (10\%) y África $(4 \%)$ continúan siendo parte de la retórica de la diplomacia cultural de México, pero poco se ha hecho realmente para ampliar su presencia en estos espacios. 
GRÁFICA 3

Regiones del mundo destinatarias de las acciones de la diplomacia cultural mexicana (2013-2018)

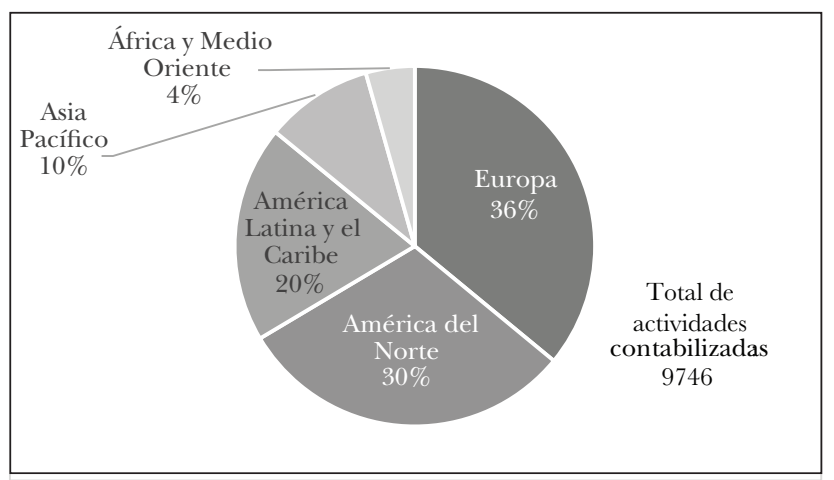

Fuente: informes anuales de la sRE.

La evaluación de las acciones culturales en el exterior que hizo la Amexcid sigue siendo uno de los temas que no se han resuelto con toda claridad. El criterio cuantitativo relativo a eventos y los asistentes a cada uno de ellos es únicamente indicativo y claramente insuficiente para entender lo que se realiza en este campo. El tema central es entender que la cultura es una cuestión relacionada con la significación y sólo en un segundo nivel asociado con el volumen de asistencia y los presupuestos. Es, en todo caso, un asunto estratégico de la política exterior, pero que, paradójicamente, carece de una estrategia clara.

Es de llamar la atención que la SRE no haya puesto más atención al proyecto de los Institutos Culturales de México en el exterior. A pesar de diversas llamadas de muchos sectores para lanzar una estrategia de largo aliento, usando los institutos como centros de atracción y provisión de información sobre México, a ninguno de los cancilleres le pareció oportuno retomar las diversas propuestas en ese sentido. Con doce sedes funcionales, algunas de ellas realmente privilegiadas (como las de Madrid, París, Guatemala y Costa Rica, 
por mencionar algunas), apenas figuran dentro de las actividades de la DGCEC. Para muchos países, como Francia, España, Italia, Japón o China, este tipo de entidades son muy importantes para el ejercicio de su diplomacia cultural.

De la misma manera, vale la pena destacar el restablecimiento de la Misión Permanente de México en la Organización de las Naciones Unidas para la Educación, la Ciencia y la Cultura (unesco), en París, tema que se había desdibujado durante el gobierno de Felipe Calderón, aduciendo razones presupuestales. En 2013, el embajador Porfirio Thierry Muñoz Ledo (miembro del SEM) asumió esa representación y le dio importancia de nuevo a ese vínculo, estratégico para la SRE y sus temas culturales. En 2016, Andrés Roemer fue asignado a dicha misión, pero tuvo una estancia de apenas unos meses y fue cesado del cargo después de mostrar una clara falta de conocimientos de las funciones diplomáticas dentro del Servicio Exterior Mexicano, al desobedecer instrucciones precisas de la canciller Claudia Ruiz Massieu. Después de esto, el embajador Federico Salas Lotfe fue nombrado en 2017 para ocupar el cargo. Los trabajos de la representación de México en la unesco fueron modestos y poco relevantes en el sexenio de Peña Nieto. Lo más importante es el apoyo que ha brindado para la inscripción de sitios mexicanos en la lista del patrimonio mundial. Por lo demás, queda la deuda de darle un lugar de reconocimiento a la presencia de México en esa institución, tan cercana a muchos mexicanos desde que la presidiera el embajador Jaime Torres Bodet hace más de sesenta años.

\section{Del conaculta a la Secretaría de Cultura}

Otro tema clave tiene que ver con la duplicidad operativa en la diplomacia cultural que se produce con la SC y las fallas de coordinación con la SRE. Conforme a lo que establece la Ley General de Cultura y Derechos Culturales, en lo relativo a la vinculación internacional, "la Secretaría de Cultura [y otras 
dependencias federales y locales] contribuirán a las acciones destinadas a fortalecer la cooperación e intercambio internacional en materia cultural, con apego a los tratados internacionales celebrados por los Estados Unidos Mexicanos y a las demás leyes aplicables en la materia"; de la misma manera, la sc es un actor primordial para la "recepción de diferentes manifestaciones culturales de otros países en la República Mexicana". ${ }^{18}$

La instancia de la SC encargada de los temas internacionales es la Dirección General de Asuntos Internacionales (DGAI), cuyo objetivo general es "coordinar, con base en el Programa Nacional de Cultura 2013-2018, las acciones de cooperación y difusión internacional con el fin de promover la cultura mexicana en todas sus expresiones, así como fortalecer el intercambio con otras naciones". ${ }^{19}$ La DGAI cuenta con dos oficinas que le permiten desarrollar sus actividades: por un lado, la Dirección de Cooperación Cultural Internacional, cuya labor es "fomentar, propiciar y dar seguimiento a proyectos de cooperación cultural internacional en materia de patrimonio cultural material e inmaterial, diversidad cultural e industrias creativas"; ${ }^{20}$ por otro, la Dirección de Difusión Cultural Internacional, con tareas enfocadas en "difundir y promover las expresiones artísticas de México en los principales foros y eventos en todo el mundo y, al mismo tiempo, acercar al público mexicano a lo más destacado del amplio horizonte de la producción artística internacional". ${ }^{21}$ Como se desprende de lo anterior, la concepción y definición conceptual de esta área es mucho más específica que su equivalente, la DGCEC de la Amexcid. Por tanto, resulta muy importante señalar que existe una evidente duplicidad y tras-

${ }^{18}$ Ley General de Cultura y Derechos Culturales, Diario Oficial de la Federación, 19 de junio de 2017, título quinto, arts. 41 y 42.

${ }^{19}$ Dirección General de Asuntos Internacionales, “¿Qué hacemos?”, México, Gobierno de la República, https://www.cultura.gob.mx/dgai/

${ }^{20}$ Loc. cit.

${ }^{21}$ Loc. cit. 
lape de funciones, que sólo se resolvió medianamente, dependiendo del criterio de los funcionarios de turno.

En el tema de la cultura en el ámbito internacional, propiamente dicho, la SC avanzó hacia varios objetivos de su competencia, pero pudo haber impulsado, con mayor vigor, un debate en beneficio de México. No hubo un liderazgo en los temas culturales a nivel internacional con la relevancia suficiente para avanzar una agenda estratégica de Estado. Ello fue claro en la poca o nula presencia de México en distintos foros multilaterales, como las Conferencias Iberoamericanas de Cultura, que en el sexenio de Peña Nieto fueron cuatro (2013, 2014, 2016 y 2018); las reuniones de la Carta Cultural Iberoamericana (2006), de la que México forma parte, o los debates sobre posicionamiento internacional al interior de la UNESCO, por ejemplo, en la serie de informes mundiales Re|Pensar las Políticas Culturales, en los que no hubo participación de ningún mexicano para reflexionar sobre estos temas.

A pesar del dinamismo que tuvo en lo internacional, México decidió ser un actor pasivo, sin entender la importancia de un rol claramente propositivo y dinámico.

Con todo, es importante mencionar que gracias al impulso del CONACULTA-SC, durante el sexenio analizado hubo dos sitios registrados como patrimonio natural, el de la Reserva de la Biósfera El Pinacate y Gran Desierto de Altar, en Sonora (2013), y el poco conocido archipiélago de Revillagigedo, en Colima (2016). Asimismo, se registró un bien cultural, el Sistema Hidráulico del Acueducto del Padre Tembleque, en Hidalgo (2015), y uno más de orden mixto, el valle de Tehuacán-Cuicatlán, hábitat originario de Mesoamérica, que se ubica entre Puebla y Oaxaca (2018). También, se inscribieron dos bienes inmateriales: "La charrería, tradición ecuestre en México" (2016) y "La romería de Zapopan: ciclo ritual de la llevada de la Virgen" (2018). Con esto, México cuenta en total con 35 sitios designados patrimonio de la humanidad (27 como bien cultural, seis como bien natural y dos como bien mixto). En lo relacionado con los bienes 
inmateriales, México ha registrado a instancias de la SC nueve tradiciones y festejos que son invaluables y aportan imaginarios positivos en el exterior, entre los que se incluye: los voladores de Papantla, el Día de Muertos, la cocina tradicional mexicana en su vertiente michoacana o el mundialmente reconocido mariachi jalisciense. Es importante hacer mención de que, como parte de la proyección de México en el exterior, el país es el séptimo con mayor cantidad de bienes inscritos en la lista de la Unesco (llevan la delantera Italia, China, España, Francia, Alemania y la India), lo que ubica a México en el primer lugar del continente americano.

\section{LA IRRELEVANCIA CULTURAL EN EL EXTERIOR: SENSIBLE DISMINUCIÓN PRESUPUESTAL}

El elefante en la sala de esta revisión, desafortunadamente, es el del presupuesto para temas culturales. Dentro de la SRE, los montos y partidas presupuestales para este rubro mantuvieron una inercia ligeramente decreciente, en comparación con el sexenio de Calderón. Aunque en lo fundamental no hubo cambios económicos significativos, la inevitable depreciación de la paridad peso-dólar, aunada a la inflación -dado que se mantuvo una constante presupuestal en pesos-, significó una reducción real en esta área. Es de recordar que el presupuesto promedio anual de la DGCEC durante el gobierno calderonista fue de 91.8 millones de pesos, de los que se destinaron 52.3 millones de pesos a la promoción y 39.5 millones de pesos a la cooperación. ${ }^{22}$ En el sexenio de Enrique Peña Nieto, la cantidad promedio por año fue de 23.9 millones de pesos para ambos rubros. Las barras del primer grupo de la gráfica 4, correspondientes a la DGCEC de la SRE, muestran la evolución presupuestal de la Amexcid en este ámbito: empieza en 2013 con un monto de 16.9 millones de pesos y termina en 2018 con 9.8 millones de pesos. El año con mayor

${ }^{22}$ César Villanueva, op. cit., p. 220. 


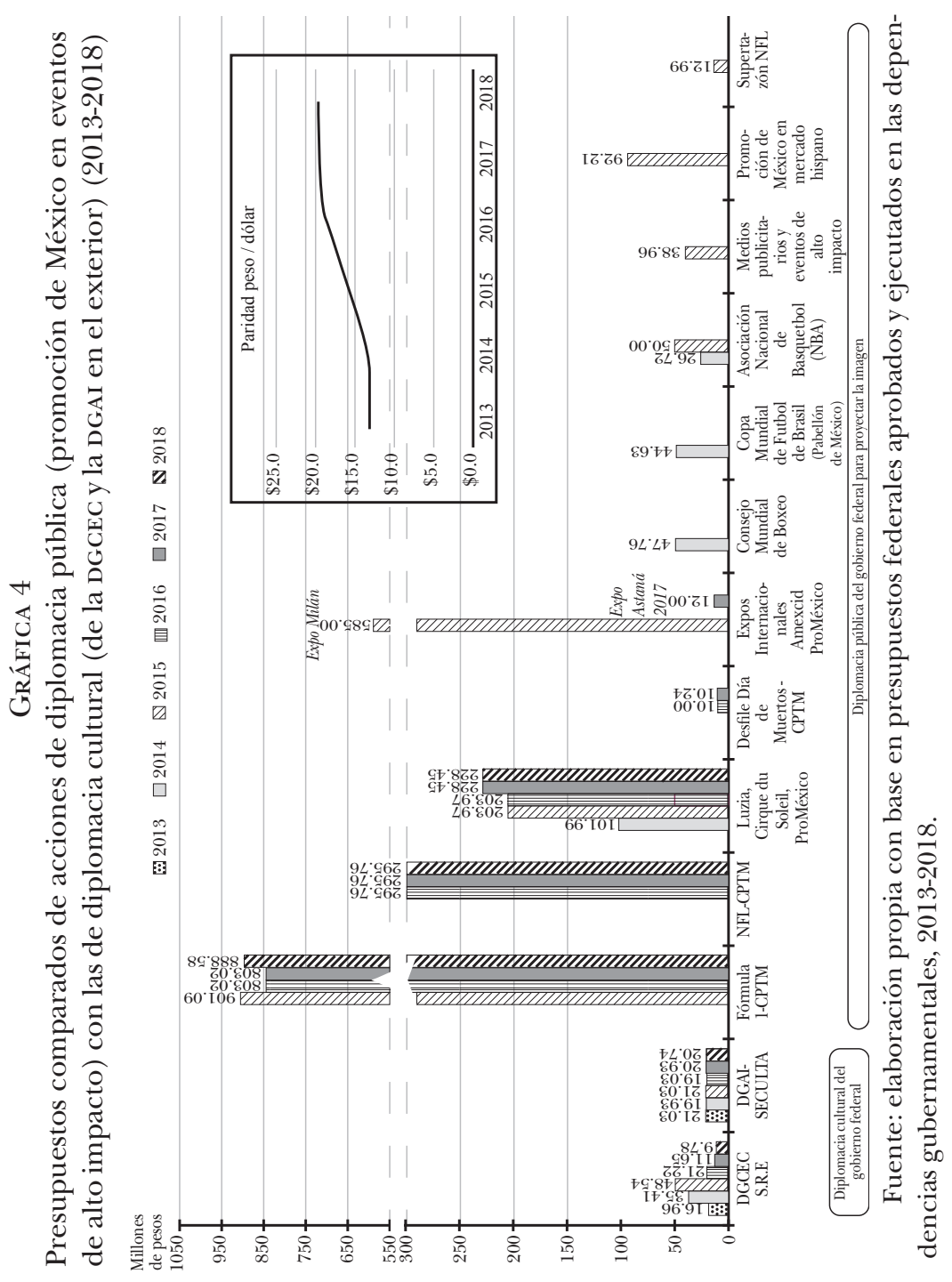


presupuesto resultó ser 2015 y el de menores recursos, 2018. El descenso presupuestal de 67.9 millones de pesos equivale a $74 \%$ de disminución real. Aunado a eso, como se observa en la gráfica siguiente, las reducciones también se relacionaron con el valor del dólar, que en diciembre de 2012 se cotizó a 12.87 pesos por unidad y alcanzó en 2018 los 20.31 pesos. Esto equivale a una devaluación de $58 \%$, pérdida que no se ajustó sistemáticamente en el presupuesto a lo largo del sexenio y que significa una disminución real en el valor económico de las actividades culturales en el exterior, dado que éstas tienen costos tasados en moneda extranjera. Este panorama es muy desolador y una señal de la poca o nula importancia que se le dio a la diplomacia cultural en el periodo de Peña Nieto.

Como también puede observarse en la gráfica 4, el presupuesto de la DGCEC (que es parte de la estructura de la Amexcid y a la vez es un órgano desconcentrado de la SRE) tiene que compararse con otras actividades paralelas en las que, como vimos antes, se fragmentó el presupuesto para el mix de la culpub diplomacy, es decir, aquella referida a promoción y cooperación cultural combinada con actividades de posicionamiento, atracción, comunicación e imagen de México en el exterior. Comencemos con el presupuesto para expos internacionales en las que participó la Amexcid y ProMéxico, a lo que se le dedicaron recursos por 598 millones de pesos para dos eventos: Milán 2015 y Astaná 2017. ProMéxico promocionó la presentación del evento escénico Luzia a nivel internacional, pagándole a la agrupación Cirque du Soleil la cantidad de 987.1 millones de pesos. ${ }^{23}$ El СРтм раtrocinó el desfile del Día de Muertos en la Ciudad de México dos años seguidos, con un costo de 20 millones de pesos. A eso hay que añadir el gasto para modificar los contenidos de

23 Agatha Pacheco, "Cirque du Solei`s Luzia is a $\$ 47.7$ million detriment to mexican artists", Seattle Weekly, 10 de mayo de 2017, https:/ / www. seattleweekly.com/arts/cirque-de-soleils-luzia-is-a-47-7-million-detrimentto-mexican-artists / 
la película Spectre, de 2015, sufragado por la Secretaría de Turismo y el gobierno de la Ciudad de México, por un monto de 278.3 millones de pesos. ${ }^{24}$

El CPTM $^{25}$ también patrocinó partidos de la NFL celebrados en México durante tres ocasiones, con un costo total de 888 millones de pesos. La misma institución patrocinó, en cuatro ocasiones, el Campeonato Mundial de Fórmula 1 de la Federación Internacional de Automovilismo, con un costo total en el sexenio de 3462000 millones de pesos, ${ }^{26}$ la mayor cifra de todos los rubros tomados en cuenta aquí. En cuanto a la promoción de la marca México en eventos de alto impacto, se erogaron 13 millones de pesos en los partidos de la NFL y 76 millones de pesos en juegos de la National Basketball Association, de EEUU, por mencionar algunos ejemplos. En el caso de la DGAI de la SC, el monto sexenal llegó a los 123 millones de pesos. Sumado esto último con el presupuesto de la DGCEC-SRE, los recursos para la diplomacia cultural en el gobierno de Peña Nieto ascienden a 267 millones de pesos. La desproporción con las otras áreas es más que evidente.

El presupuesto para diplomacia cultural de México en el sexenio 2013-2018 fue liliputense, comparado con lo que dedican países como España (2013 = 102 millones de dólares, sin contar AECID $),{ }^{27}$ Australia $(2017=230$ millones de dólares, sin contar diplomacia pública ni AOD) o Corea del Sur

${ }^{24}$ Ben Beaumont-Thomas, "Mexico City estimates $\$ 24 \mathrm{~m}$ loss due to filming of latest James Bond movie", The Guardian, 2 de abril de 2015, https://www.theguardian.com/film/2015/apr/02/mexico-city-24m-lossjames-bond-shoot-spectre

${ }^{25}$ El financiamiento para estos eventos por parte del gobierno federal se obtiene de los derechos que pagan los turistas por visitar México (Ley Federal de Derechos, Diario Oficial de la Federación, 31 de diciembre de 1981, art. 18-A.

${ }^{26}$ Raúl Ochoa, "Fórmula 1: dinero público, negocio privado", Proceso, 2 de agosto de 2014, https://www.proceso.com.mx/378714/formula1-dinero-publico-negocio-privado

27 "E. Public Funding of Culture", Compendium, cultural policies \& trends (sitio de internet), http://www.culturalpolicies.net/web/statisticsfunding.php 
(2018 = 260 millones de dólares), por no mencionar presupuestos para asuntos culturales en el exterior como el de Francia $^{28}(2016=840$ millones de dólares, sin contar la Alliance Française y la promoción turística), Estados Unidos ${ }^{29}$ (2016 = 591 millones de dólares, sin contar diplomacia pública), Reino Unido ${ }^{30}$ (2017 = 207 millones de dólares, sin contar con el British Council) o Japón ${ }^{31}$ (2016 = 430 millones de dólares) .

Finalmente, la dispersión del presupuesto para diplomacia cultural y áreas afines (diplomacia pública, marca país, turismo cultural, cooperación cultural, etc.) en diversas áreas del gobierno es una de las notas más significativas. La fragmentación de estrategias y esfuerzos institucionales, minimizó las posibilidades de hacer de la diplomacia cultural la estrategia central de la política exterior del Estado mexicano en esos temas.

\section{LA IMAGEN DE MÉxico EN EL MUNDo: 2013-2018}

México no puede obviar el hecho de que una imagen-país favorable es un prerrequisito, en el ámbito interno, para la

28 Sophie Heyligers, "Comparative research. The Cultural Policy towards China of the Netherlands Compared to Denmark, France, Germany \& the uk", Dutch Culture, Amsterdam, 2016, https:/ / dutchculture. $\mathrm{nl} /$ sites/default/files/atoms/files/Comperative\%20Research\%20-\%20 FINAL\%20REPORT_0.pdf ; "Projet de loi de finances rectificative pour 2015", República Francesa, 2015, https://www.performance-publique. budget.gouv.fr/sites/performance_publique/files/farandole/ressources/2015/lifipdf/PLFR_2015.pdf

${ }^{29}$ United States Advisory Commission on Public Diplomacy, op. cit.; Thomas Lum (coord.), "Comparing Global Influence: China's and us Diplomacy, Foreign Aid, Trade, and Investment in the Developing World", https://fas.org/sgp/crs/row/RL34620.pdf

${ }^{30}$ Foreign \& Commonwealth Office, "Annual Report and Accounts 2016-17”, https://assets.publishing.service.gov.uk/government/uploads/ system/uploads/attachment_data/file/625437/FCO-annual-report2017-body-print-ready.pdf

31 "Efforts to Promote Understanding of and Trust in Japan", en Japan's Foreign Policy to Promote National and Worldwide Interests, Diplomatic Bluebook, 2016, https://www.mofa.go.jp/files/000177726.pdf 
gobernabilidad y, en el exterior, para la reputación. La ruta para apuntalar con éxito una imagen internacional favorable no es un misterio. Los factores externos de una imagenpaís favorable se traducen en tres aspectos: capacidad de atracción, capacidad de influencia y persuasión, y adquisición de una reputación. En otras palabras, ésta es la estrategia que se conoce como poder suave. ${ }^{32}$ Para México, la atracción se traduce en el incremento de los flujos de capital, de personas -especialmente turismo y migración- y de industrias que, a su vez, son fuentes de empleo. La capacidad de influencia y persuasión se da particularmente en el ámbito diplomático, bilateral y multilateral, así como en las negociaciones sobre temas de interés de la nación, en el ámbito del comercio, el turismo, el entretenimiento, el arte o de la cultura en su visión más profunda. La reputación es un intangible simbólico que se relaciona con la percepción de confiabilidad y legitimidad de las acciones del Estado, lo que se traduce en respeto y admiración. ${ }^{33}$

Es preciso subrayar que la responsabilidad de la reputación y la imagen de México en el exterior corresponde a los funcionarios de la SRE por ley y es en ese espacio donde estos temas deben ser pensados, instituidos e instrumentados. De hecho, la Ley del Servicio Exterior Mexicano define las "actividades diplomáticas" como "las acciones desarrolladas por servidores públicos en el ámbito de las relaciones internacionales de México, con la finalidad de cumplir con los objetivos de la política exterior e incrementar la presencia, el impacto y prestigio de México en el mundo". ${ }^{34}$

En el sexenio de Peña Nieto, la imagen internacional de México ha sido señalada como uno de los problemas centrales que enfrentó la política exterior del país. Diversas fuentes

${ }^{32}$ César Villanueva, "Construyendo el poder suave de México", Revista Mexicana de Política Exterior, núm. 111 (2017), pp. 5-19.

${ }^{33}$ Leonardo Curzio, Orgullo y prejuicios: reputación e imagen de México, México, unam, 2016.

${ }^{34}$ Ley del Servicio Exterior Mexicano, Diario Oficial de la Federación, 4 de enero de 1994, art. 1 bis y art. 2, fracc. VI, VIII y IX. 
confirman esta preocupación. En el estudio realizado en 2016, La imagen de México en el mundo, ${ }^{35}$ se asumió que ésta se presenta bajo tres tipologías distintivas, pero contrastantes: la primera es la de un país dependiente, que representa los lastres de la desigualdad social, la corrupción, las disfuncionalidades graves del Estado de derecho, las instituciones de seguridad muy endebles, así como de un poder suave tímido y acotado. En contraste, la segunda es la de un país emergente que da señales inequívocas de progreso económico, estabilidad financiera, desarrollo urbano, libertades cívicas y un dinamismo diplomático centrado en algunas agendas de relevancia internacional, en la cual también aparece un apoyo importante a las artes y la cultura. Finalmente, la tercera tipología ubica al país en el exterior como una nación exótica, de la que se desprenden representaciones como sombreros, mariachis, tequila, aztecas, Frida Kahlo o el Chavo del Ocho, entre otras. Estos últimos signos de alteridad son parte de un "folklore mexicano" o "rareza nacional" que generan estereotipos y estigmas simbólicos que, a pesar de su relativa inocencia o atractivo, terminan afectando negativamente al país, más que beneficiándolo.

En el renglón de percepción e imagen internacional, el mandato de Peña Nieto se inaugura con una campaña muy visible y ostentosa organizada por el embajador Diego Gómez Pickering, conocida como el "momento mexicano" (MeMo, Mexican Moment). Dicha campaña logró cambiar de manera temporal la manera en que los medios internacionales se referían a México, después de la crisis vivida durante el gobierno de Calderón. ${ }^{36}$ Sin embargo, después de septiembre de 2014, la situación se deterioró muy rápido en el campo de la violencia y la corrupción, y la estrategia del MeMo resultó insuficiente. La imagen del país en el exterior sufrió un revés

35 César Villanueva, La imagen de México en el mundo, México, Universidad Iberoamericana, 2016.

36 "Interview with Mexico's Ambassador to the UK, Diego Gomez Pickering", LatAm Investor, 20 de marzo de 2014, http://latam-investor. com/2014/03/interview-mexicos-ambassador-uk-diego-gomez-pickering/ 
del cual no pudo recuperarse. En el análisis realizado de doce medios internacionales, se encontró que en el período de 2013 a 2018 se emitieron 7412 noticias sobre México, de las cuales el $56 \%$ fueron clasificadas como negativas y únicamente $17 \%$ fueron claramente positivas. Los temas más perjudiciales para México fueron indudablemente el del crimen contra los estudiantes de la normal de Ayotzinapa, Guerrero, en 2014; la corrupción asociada al presidente Peña Nieto, especialmente en el caso de la "casa blanca"; la delincuencia organizada y la violencia resultante, en particular el desarrollo de lo que se conoce como "narcoviolencia", así como la violación sistemática de los derechos humanos en México, incluidos los asesinatos de periodistas, que se incrementaron de manera alarmante en el sexenio. Los temas más positivos fueron los asociados con las muchas expresiones de la cultura mexicana, la diversidad de la oferta turística, las oportunidades de inversión en el país y, de alguna forma, el posicionamiento de figuras mexicanas en el exterior, en especial en el cine de Hollywood. Las redes sociodigitales castigaron duramente la gestión de Peña Nieto y las estrategias de comunicación en ese rubro resultaron inoperantes para contrarrestar la cantidad de expresiones negativas. De un análisis de 9790 tuits emitidos desde el exterior durante ese sexenio, se concluye que $76 \%$ eran expresiones negativas contra México, los mexicanos y, muy especialmente, contra su(s) gobierno(s). Las alertas de viaje internacionales de nueve países que se analizaron para el estudio mencionado ${ }^{37}$ muestran que en $72 \%$ de los casos los mensajes asociados con la asesoría y las advertencias eran negativas hacia el país.

La percepción internacional global que se tuvo del país en el sexenio estudiado es preocupante, particularmente por el desarrollo de un estereotipo que identifica a México como una nación "peligrosa”, "disfuncional” o, en el peor de los

${ }^{37}$ Los datos fueron obtenidos entre 2013 y 2017 de las páginas oficiales de los ministerios de asuntos exteriores del Reino Unido, Canadá, Estados Unidos, España, Italia, Japón, Corea, Francia y Suecia. 
casos, como un "Estado fallido". Dicho estereotipo ya es evidente en algunas series televisivas estadounidenses de circulación mundial, en algunas películas sobre temas mexicanos, en las referidas alertas de viaje y, muy específicamente, en algunas expresiones públicas denigrantes, como las que el presidente de Estados Unidos, Donald Trump, hizo desde su precampaña y a lo largo de los dos años de su régimen, frente a lo cual el gobierno no tuvo una estrategia defensiva ni de contención de daños.

\section{Conclusiones}

En términos analíticos, la cultura es el quid de la diplomacia, es su esencia y sustancia; es, en muchos sentidos, lo que la hace posible. Estrictamente hablando, lo diplomático no es posible sin una plataforma cultural, que es su complemento inherente, dado que no puede aspirarse a entender el mundo y sus actores sino a través de códigos culturales, sean éstos lingüísticos, narrativos o simbólicos. Sólo después, todo lo demás.

La diplomacia cultural tendría que conceptualizarse de manera más amplia, atendiendo a lo que ha ocurrido en el mundo de las relaciones internacionales y la cultura global en los últimos años. Es un concepto de una enorme actualidad que refleja la complejidad de una profesión que tiene prácticas específicas. En un primer nivel, la diplomacia cultural puede comprenderse como agente de la cooperación (intercambios académicos, colaboración técnica-científica y becas) y la promoción de la cultura (exposiciones, eventos, impulso del español, etc.), siguiendo los principios de política exterior. En un segundo nivel, puede ser una estrategia con una visión cosmopolita y más universalista: "la comprensión de otras culturas y el fomento de la paz a nivel internacional”. Finalmente, es importante que la sRE defina con claridad las responsabilidades que se derivan de la cooperación cultural, que es más que la mera promoción, y que a la 
fecha no caben muy bien en su organigrama y sufren la presión de la sc, que quisiera manejar la promoción cultural internacional y los Institutos Culturales de México en el exterior.

Es importante que la cancillería tenga el papel de operador y estructurador de los esfuerzos que en diplomacia cultural realizan diversos agentes interesados: la Sc, la Secretaría de Educación Pública, el Consejo Nacional de Ciencia y Tecnología, la Secretaría de Turismo, los canales 11 y 22 de televisión, Radio Educación, universidades, organismos no gubernamentales, empresas y fundaciones culturales, y gobiernos locales. La SRE debe desarrollar una estrategia más sólida de diplomacia pública, cuya principal tarea es la de comunicarse directamente con los públicos exteriores. Puede construirse un "poder suave a la mexicana", basado en sus tres principales fuentes a nivel cultural, así como en la cooperación y otros rubros específicos de la diplomacia multilateral, sobre todo. Hay otras fuentes más particularmente influyentes en el mundo de habla hispana, como la televisión, la música y el cine (que también son cultura). Otras más que no han sido explotadas estratégicamente, como por ejemplo, la gastronomía, la enseñanza del español como segunda lengua y las industrias creativas, a través de un instituto de clase mundial que sea un escaparate de la identidad y los productos culturales mexicanos, en el que el Fondo de Cultura Económica, como institución cultural añeja, debiera ser una punta de lanza.

También asombran tres hallazgos de gran trascendencia: primero, el país hace un uso muy modesto de su capacidad cultural, vista como un elemento clave de su poder suave y que, de forma colateral, le permite avanzar hacia sus objetivos de política exterior; segundo, la marca México tiene una significación exótica confusa, que causa controversias en los públicos externos y está relegada principalmente a asuntos económicos y de turismo, desaprovechándose así el potencial de la diplomacia pública en la comunicación de temas sociales y políticos de relevancia para las sociedades de otros países. Tercero, en general, la investigación realizada evidencia que ha habido una falta de coordinación federal para 
trazar una estrategia sólida y duradera en cuanto a la imagen de México en el mundo, como parte de una política de Estado. Por supuesto, esta idea no excluye el trabajo multidisciplinario e interinstitucional, pero sorprende que durante el sexenio de Peña Nieto el tema se fragmentara inexplicablemente, finalizando, como se ha visto, en manos de al menos cinco instancias del gobierno federal. Más allá de los resultados, la estrategia debió ser, por derecho y por ámbito de especialización, una prerrogativa de la SRE.

\section{REFERENCIAS BIBLIOGRÁFICAS}

Beaumont-Thomas, Ben, "Mexico City estimates $\$ 24 \mathrm{~m}$ loss due to filming of latest James Bond movie", The Guardian, 2 de abril de 2015.

Consejo de Promoción Turística de México, Primer informe de logros Programa Institucional 2014-2018, Ciudad de México, CPTM, 2014.

Corona, César, "1. Un proyecto de 485 millones de pesos sin pies ni cabeza" (blog personal), 17 de enero de 2106, https://cesarcorona.exposure.co/intro-mexico-expo2015

Cull, Nicholas, "El futuro de la diplomacia pública: implicaciones para México", Revista Mexicana de Política Exterior, núm. 96 (2012), pp. 45-73.

Curzio, Leonardo, Orgullo y prejuicios: reputación e imagen de México, México, unam, 2016.

Decreto por el que se reforman, adicionan y derogan diversas disposiciones del Reglamento de la Oficina de la Presidencia de la República, Diario Oficial de la Federación, 30 de abril de 2015.

Dirección General de Asuntos Internacionales, “¿Qué hacemos?", México, Gobierno de la República, https://www.cultura.gob. $\mathrm{mx} /$ dgai/

"E. Public Funding of Culture", Compendium, cultural policies \& trends (sitio de internet), http://www.culturalpolicies.net/web/ statistics-funding.php

"Efforts to Promote Understanding of and Trust in Japan", en Japan's Foreign Policy to Promote National and Worldwide Interests, 
Diplomatic Bluebook, 2016, https://www.mofa.go.jp/files/00017 7726.pdf

Foreign \& Commonwealth Office, "Annual Report and Accounts 2016-17”, https://assets.publishing.service.gov.uk/government /uploads/system/uploads/attachment_data/file/625437/ FCO-annual-report-2017-body-print-ready.pdf

Heyligers, Sophie, "The Cultural Policy towards China of the Netherlands Compared to Denmark, France, Germany \& the uK", Dutch Culture, Amsterdam, 2016, https://dutchculture. $\mathrm{nl} /$ sites/default/files/atoms / files/Comperative\% $20 \mathrm{Re}$ search \%20-\%20FINAL\%20REPORT_0.pdf

"Interview with Mexico's Ambassador to the Uk, Diego Gomez Pickering”, LatAm Investor, 20 de marzo de 2014, http:/ /latam-in vestor.com/2014/03/interview-mexicos-ambassador-uk-die go-gomez-pickering/

KizLARI, Dimitra y Kalliopi Fouseki, "The Mechanics of Cultural Diplomacy: A Comparative Case Study Analysis from the European Context", The Journal of Arts Management Law and Society, vol. 48, núm. 2 (2017), pp. 133-147.

Ley del Servicio Exterior Mexicano, Diario Oficial de la Federación, 4 de enero de 1994.

Ley Federal de Derechos, Diario Oficial de la Federación, 31 de diciembre de 1981.

Ley General de Cultura y Derechos Culturales, Diario Oficial de la Federación, 19 de junio de 2017.

Lum, Thomas (coord.), "Comparing Global Influence: China's and us Diplomacy, Foreign Aid, Trade, and Investment in the Developing World", https://fas.org/sgp/crs/row/RL34620.pdf

OchoA, Raúl, "Fórmula 1: dinero público, negocio privado", Proceso, 2 de agosto de 2014, https:/ / www.proceso.com.mx/378714/ formula-1-dinero-publico-negocio-privado

Olivares, Karina, Diplomacia cultural de México en el periodo 19881994: el caso de "México, Esplendores de treinta siglos", Ciudad de México, unAm, 2005.

Pacheco, Agatha, "Cirque du Soleil's Luzia is a \$47.7 million detriment to mexican artists", Seattle Weekly, 10 de mayo de 2017, 
https:/ / www.seattleweekly.com/arts/cirque-de-soleils-luzia-isa-47-7-million-detriment-to-mexican-artists /

Plan Nacional de Desarrollo 2013-2018, Diario Oficial de la Federación, 20 de mayo de 2013.

"ProMéxico. México participará en Expo Mundial Astaná 2017", 1 de junio de 2017, Comunicado 39/2017, 1 de junio de 2017, http://www.promexico.gob.mx/es/mx/comunicado-39-17

ProMéxico, “¿Qué hacemos?”, México, Gobierno de la República, https://www.gob.mx/promexico/que-hacemos

Programa de Cooperación Internacional para el Desarrollo 2014-2018, Diario Oficial de la Federación, 30 de abril de 2014, http:/ / www.dof.gob.mx/nota_detalle.php?codigo=5342827\&f echa $=30 / 04 / 2014$

"Projet de loi de finances rectificative pour 2015", República Francesa, 2015, https://www.performance-publique.budget.gouv. $\mathrm{fr} /$ sites/performance_publique/files/farandole/ressources/ 2015/1fi/pdf/PLFR_2015.pdf

Reglamento Interior de la Secretaría de Relaciones Exteriores, Diario Oficial de la Federación, 8 de enero de 2009.

Schneider, Cynthia, "Cultural diplomacy: hard to define, but you'd know it if you saw it”, Brown Journal of World Affairs, vol. 13, núm. 1 (2006), pp. 191-203.

Tadeo, Eduardo, "Public Diplomacy, Soft Power and Language: The Case of the Korean Language in Mexico City", Journal of Contemporary Eastern Asia, 17 (2018), pp. 27-49.

United States Advisory Commission on Public Diplomacy, "2017 Comprehensive Annual Report on Public Diplomacy \& International Broadcasting", https://www.state.gov/documents/ organization/274950.pdf

Villanueva, César, "Construyendo el poder suave de México", $R \ell^{-}$ vista Mexicana de Política Exterior, núm. 111 (2017), pp. 5-19.

Villanueva, César, "Crónica de un declive anunciado: la diplomacia cultural en el sexenio de Felipe Calderón”, Foro Internacional, Vol. 53 núms. 3-4 (2013), pp. 845-872.

Villanueva, César, La imagen de México en el mundo, México, Universidad Iberoamericana, 2016. 